\title{
The role of synchrotron self-absorption in the late radio emission from SN 1993J
}

\author{
M. A. Pérez-Torres ${ }^{1, \star}$, A. Alberdi ${ }^{2}$, and J. M. Marcaide ${ }^{1}$ \\ 1 Departamento de Astronomía y Astrofísica, Universidad de Valencia, 46100 Burjassot, Valencia, Spain \\ e-mail: J.M.Marcaide@uv.es \\ 2 Instituto de Astrofísica de Andalucía, CSIC, Apdo. Correos 3004, 18080 Granada, Spain \\ e-mail: alberdi@laeff.esa.es
}

Received 14 November 2000 / Accepted 23 May 2001

\begin{abstract}
The standard model for radio supernovae considers that the observed synchrotron radio emission arises from the high-energy shell that results from the strong interaction between the expanding supernova ejecta and the circumstellar medium. This emission is considered to be only partially absorbed by ionized thermal electrons in the circumstellar wind of the progenitor star. Based on a study of the radio light curves of the type II supernova SN1993J, we present evidence of synchrotron self-absorption. Our modeling of the radio light curves requires a large initial magnetic field, of about 30 Gauss, and the existence of an (initially) highly-relativistic population of electrons. We also show that while at early epochs the dominant absorption mechanism is external absorption by thermal electrons, at late epochs and long wavelengths the dominant absorption mechanism is synchrotron self-absorption. Consequently, the spectral turnover takes place at much shorter wavelengths than expected in the standard model, and at long wavelengths ( $\geq 90 \mathrm{~cm}$ at current epochs) the flux predictions depart substantially from those of the standard model.
\end{abstract}

Key words. radiation mechanisms: non-thermal radiative transfer - supernovae: general supernovae: individual (SN 1993J)

\section{Introduction}

Radio emission from supernovae has been previously interpreted in terms of an optically-thin shell, whose emission is partially suppressed by external free-free absorption (e.g., Chevalier 1982a; Weiler et al. 1989, 1990). In this model - known as the standard interaction model (Chevalier 1982a), hereafter SIM - a strong interaction between the expanding supernova ejecta $\left(\rho_{\mathrm{ej}} \propto r^{-n}\right)$ and the circumstellar medium $\left(\rho_{\mathrm{cs}} \propto r^{-s}\right)$ is expected. In the SIM, this interaction causes the formation of a selfsimilarly expanding $\left(R_{\mathrm{sh}} \propto t^{m} ; m=(n-3) /(n-s)\right)$ shelllike structure (Chevalier 1982b; Nadyozhin 1985), from which the observed synchrotron radio emission arises. In addition, it is assumed (Chevalier 1982a) that (i) both magnetic energy density, $\epsilon_{B} \propto B^{2}$, and relativistic energy density, $\epsilon_{\text {rel }} \propto N_{0} E^{2-p}$, evolve with time as the post-shock thermal energy density $\left(\propto \rho_{\mathrm{sh}} v_{\mathrm{sh}}^{2}\right)$; (ii) the synchrotron

Send offprint requests to: M. A. Pérez-Torres,

e-mail: torres@ira.bo.cnr.it

* Present address: Istituto di Radioastronomia, Via P. Gobetti 101, 40129 Bologna, Italy. emission is optically thin; and (iii) the external absorbing medium has a power-law dependence $s=2$.

The discovery of SN 1993J (García 1993) on March 28, 1993 in the galaxy M 81, brought an unprecedented opportunity to test the SIM for supernovae. The early discovery of hydrogen lines in its spectra (Fillipenko \& Matheson 1993) classified this supernova as Type II. Marcaide and coauthors showed the radio emission to arise from a shelllike structure (Marcaide et al. 1995a) that expands selfsimilarly (Marcaide et al. 1995b), thus giving strong support to the SIM. Marcaide et al. (1997) also reported on the deceleration of the expansion $(m=0.86 \pm 0.02)$, and concluded that there is strong evidence for the circumstellar medium density profile to be of the form $\rho_{\mathrm{cs}} \propto r^{-s}$, with $s=1.66$, in good agreement with previous modeling of radio observations ( $s=1.5$; Van Dyk et al. 1994; Lundqvist 1994) and with the modeling of both radio and X-ray observations, which require $1.5 \lesssim s \lesssim 1.7$ (Fransson et al. 1996; hereafter FLC96). Attempts to fit the radio light curves of SN 1993J using the SIM have been unsuccessful so far, and have led several authors (Van Dyk et al. 1994; Lundqvist 1994, FLC96; 
Marcaide et al. 1997) to consider alternative power-law profiles for the circumstellar medium around SN 1993J, or to include synchrotron self-absorption as an additional absorbing mechanism (Fransson \& Björnsson 1998, hereafter FB98).

\section{Synchrotron radio emission from SN 1993J}

In an attempt to gain insight into the physical conditions of SN 1993J, and also motivated by our VLBI monitoring of this unique radio supernova, we have written a numerical code (Pérez-Torres 1999) that computes the synchrotron radio emission from an expanding Type II supernova. Although our code will be fully described elsewhere, we briefly outline it here. We solve the synchrotron radiative transfer equation throughout the shell (including synchrotron emission and synchrotron self-absorption), and take into account both synchrotron and expansion losses. We assume a (modified) SIM scenario, in which the supernova synchrotron radio emission comes from the interaction shell that forms shortly after shock breakout. (The SIM scenario is modified in the sense that we do not assume the synchrotron emission to be optically thin.) We assume the emission to be due to the existence of relativistic electrons, $N(E, r)$, orbiting in a random magnetic field, $B(r)$. We assume an initially injected relativistic electron distribution with a power-law energy dependence, $N(E, r)=N_{0}(r) E^{-p}$, and also assume a continuous reacceleration of those electrons.

In modeling the radio emission from SN 1993J, we take into account all available observational data: (i) the distance to SN1993J, $D=3.6 \mathrm{Mpc}$ (Freedman et al. 1994); (ii) the supernova explosion date, $t_{\exp }=$ March 28.0, 1993 (Nomoto et al. 1993; Podsiadlowski et al. 1993; Bartunov et al. 1994); (iii) the supernova deceleration parameter, $m=0.86$ (Marcaide et al. 1997); (iv) the width of the radio emitting shell, $\Delta R=0.3 R_{\text {shock }}$ (Marcaide et al. 1995b, 1997); (v) the index of the circumstellar density material, $s=1.66$ (Marcaide et al. 1997). Finally, we assume (vi) the same external electron temperature profile as in FB98: $T_{\mathrm{e}}(r)=\max \left[T_{15}\left(10^{15} \mathrm{~cm} / r\right), 2 \times 10^{5} \mathrm{~K}\right]$, where $T_{15}$ is the electron temperature in the circumstellar medium at $r=10^{15} \mathrm{~cm}$, which in our model is equal to $1.7 \times 10^{6} \mathrm{~K}$. The thermal electron density of the circumstellar medium (assumed to be fully ionized with abundances $X=0.73, Y=0.23$ ), is given by $n_{\mathrm{cs}}=1.27 \times 10^{8} M_{\mathrm{W}}\left(r / 6.35 \times 10^{14} \mathrm{~cm}\right)^{-s} \mathrm{~cm}^{-3}$, where we have introduced the wind parameter $M_{\mathrm{W}} \equiv$ $\left(\dot{M} / 10^{-5} M_{\odot} \mathrm{yr}^{-1}\right)\left(v_{\mathrm{w}} / 10 \mathrm{~km} \mathrm{~s}^{-1}\right)^{-1} \mathrm{~g} \mathrm{~cm}^{-1}$, and $\dot{M}$ and $v_{\mathrm{w}}$ are the mass-loss rate and velocity, respectively, of the presupernova wind. As in the SIM, we assume that both $B$ and $N_{0}$ evolve with time as the post-shock thermal energy density, $\rho_{\mathrm{sh}} v_{\mathrm{sh}}^{2}$. With $m=0.86$ and $s=1.66$ from our VLBI observations, we then obtain the following evolution laws for magnetic field and relativistic particles, respectively: $B \propto r^{1-s / 2-1 / m}=r^{-0.99}, N_{0} \propto$ $r^{2(1-s / 2-1 / m)}=r^{-1.99}$, that is, $B \propto r^{-1}$, and $N_{0} \propto r^{-2}$.

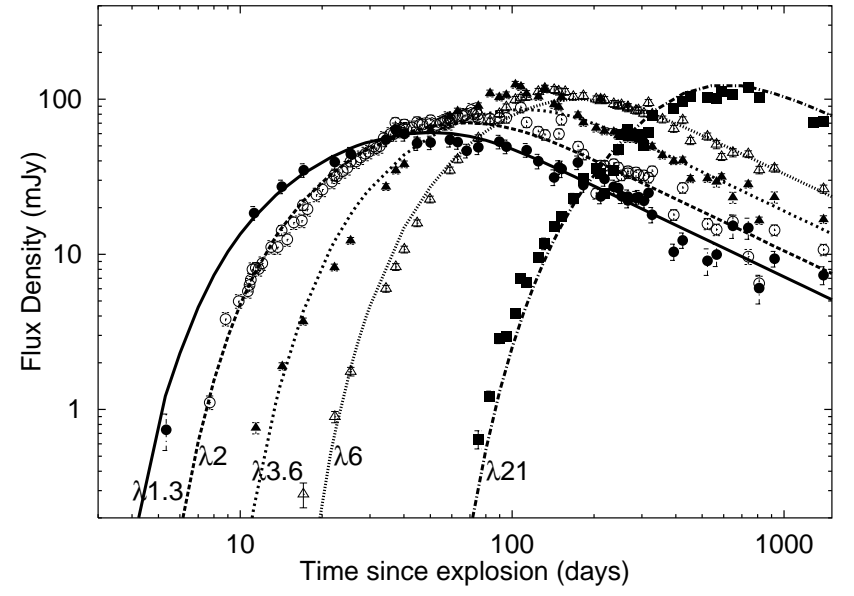

Fig. 1. Radio light curves for SN 1993J at $\lambda 1.3 \mathrm{~cm}$ (filled circles), $\lambda 2 \mathrm{~cm}$ (open circles), $\lambda 3.6 \mathrm{~cm}$ (filled triangles), $\lambda 6 \mathrm{~cm}$ (open triangles), and $\lambda 21 \mathrm{~cm}$ (filled squares), from measurements made using the VLA (Van Dyk et al. 1994; Van Dyk, private communication) and, at $2 \mathrm{~cm}$, also the Ryle Telescope (Pooley \& Green 1993). The lines represent our best fit model as described in the text.

These scalings correspond to those in model 4 of Chevalier (1996), which give the slowest evolution of $B$ and $N_{0}$, and therefore of the radio emission from SN1993J. The other three models consider a dependence of $B \propto t^{-1}$, or $N_{0} \propto t^{-2}$ or both.

We model the available radio data using the following parameters: (1) the spectral index of the injected electron distribution, $p ;(2)$ the initial magnetic field, $B\left(r_{0}\right)$; (3) the initial value of the injected function of relativistic electrons, $N_{0}\left(r_{0}\right)$; and (4) a low-energy cut-off for the relativistic electrons, $\gamma_{\min }\left(r_{0}\right) \equiv E_{\min }\left(r_{0}\right) / m_{\mathrm{e}} c^{2}$. We fit the above parameters at the reference radius $r_{0}=6.35 \times 10^{14} \mathrm{~cm}$, corresponding to the outer shell radius at our first epoch $\left(t_{0}=\right.$ 2.34 days). We find that the multi-wavelength radio data for SN 1993J (Fig. 1) are best reproduced by a model with $p=3, B\left(r_{0}\right)=27 \mathrm{G}, N_{0}\left(r_{0}\right)=6.7 \times 10^{-7} \mathrm{erg}^{p-1} \mathrm{~cm}^{-3}$, and $\gamma_{\min }\left(r_{0}\right) \approx 90$.

We estimate the uncertainties in the parameters of our model by modifying each of the best-fit parameters (one at a time), and comparing via a least-squares fit the resultant emission with that predicted by our best-fit model. These calculations show the extreme sensitivity of our results to $p$, which needs to be kept very close to 3 (within a small few per cent) to obtain any meaningful fit to the radio light curves. The other three parameters are less well constrained, and a $20 \%$ variation of each of their bestfit values result in reasonable fits. In addition, we have considered the existence of couplings between some of the parameters, especially $N_{0}$ and $B$. Further testing shows that factor of two variations in each of the three poorlyconstrained parameters give very poor fits to the observations. Therefore, we adopt factor of two modeling errors for each of those three parameters. 


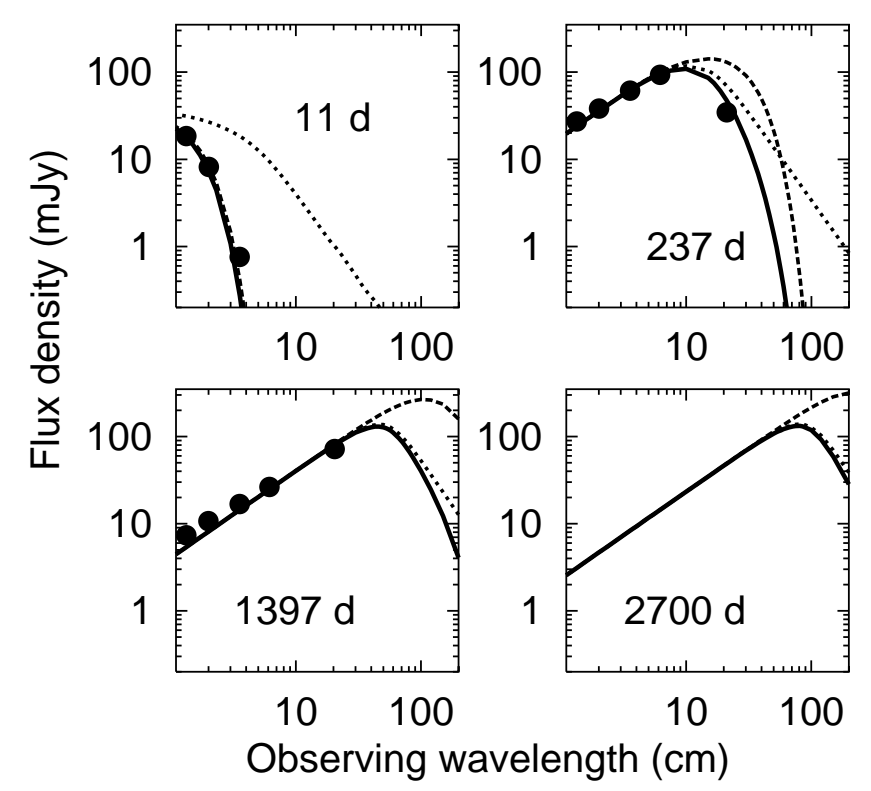

Fig. 2. Fits to spectra at 11, 237, and 1397 days after explosion, and predicted spectrum at 2700 days, considering external free-free absorption (dashed lines), synchrotron self-absorption (dotted lines), and both external free-free absorption and synchrotron self-absorption (solid lines). The filled circles represent the available observational data for each epoch. At early epochs, the radio spectrum of SN 1993J (upper left plot) is well explained by the solely effect of free-free absorption from the external medium. At late epochs (lower left plot), however, both free-free absorption and synchrotron self-absorption predict a similar spectral behavior for the observed wavelength range, but a different one at wavelengths around and above $\lambda 40 \mathrm{~cm}$, at which observations are not available. The lower right-hand plot shows the predicted spectrum at current epochs. This prediction can be tested to discriminate between the (external) free-free and (internal) self-absorption mechanisms described in the text.

\subsection{Spectral evolution of SN1993J: evidence for synchrotron self-absorption}

Our simulated spectra for SN 1993J agree well with the observed spectra (Fig. 2). The supernova spectrum changes from a mainly inverted spectrum at early epochs to a more typical optically thin spectrum at late epochs and for an increasingly wide wavelength range. As shown in Fig. 2, external free-free absorption is dominant at early times $(t \leq 100$ days), but synchrotron self-absorption becomes increasingly important with time for the long-wavelength radio emission. Thus, the standard interaction model is incomplete, at least for SN 1993J, in that it ignores synchrotron self-absorption, which according to our modeling is the most relevant absorption mechanism - as the supernova ages - for the longer wavelengths. At current epochs, our model predicts that the flux at $\lambda 90 \mathrm{~cm}$ is $\sim 120$ mJy for the synchrotron self-absorption dominated scenario, and $\sim 200$ mJy for the free-free absorption dominated scenario. This prediction - which would represent a direct test to discern the relevant absorption mechanism for SN 1993J - can be checked using $\lambda \sim 90 \mathrm{~cm}$ observations of SN 1993J with a sensitive radio interferometer.

\section{Results and discussion}

Our modeling results show that a combination of synchrotron self-absorption and external free-free absorption can reproduce well the evolution of the radio light curves of SN 1993J. Therefore, the ad hoc external inhomogeneous medium ("clumpy external absorption") previously invoked by Van Dyk et al. (1994) to model the radio light curves seems to be unnecessary. We have also considered the Razin-Tsytovich effect (the suppression of the emission below a certain wavelength due to the existence of a plasma) as an alternative mechanism to free-free absorption. However, the densities of thermal electrons required for the Razin-Tsytovich mechanism to be effective are unrealistically large. For example, at 11.5 days, we find that the number density of thermal electrons should be larger than $\sim 1.3 \times 10^{9} \mathrm{~cm}^{-3}$ for the Razin-Tsytovich effect to be important. This value is about an order of magnitude larger than needed to correctly model the X-ray luminosity at those epochs (FLC96). The same holds at late epochs, and we therefore rule out the Razin-Tsytovich effect as a plausible alternative to external free-free absorption. We point out here that the index of the circumstellar medium density profile, $s=1.66$, was obtained by Marcaide et al. (1997) under the assumption that external free-free absorption was the only absorption mechanism acting in SN1993J. Although there is increasing evidence that synchrotron self-absorption is also relevant for SN 1993J (Chevalier 1998, FB98, this work), we note that at the early epochs used by Marcaide et al. (1997) free-free absorption is the dominant mechanism, while synchrotron self-absorption can be neglected (see Fig. 2, epoch 11 days). We have tried to fit the light curves using either an standard index for the circumstellar wind $(s=2)$, or a much flatter one $(s=1.5)$ which would still satisfy the range of $1.5 \lesssim s \lesssim 1.7$ inferred from X-ray observations (FLC96). As Fig. 3 shows, an $s=2$ wind causes a fall-off of the observed emission which is far too rapid. On the contrary, a wind with $s=1.5$ makes the radioemission to fall-off too slowly. For the case $s=2$, we also investigated different scalings for $B$ and $N_{0}$; namely, we used the other three scalings mentioned by Chevalier (1996). However, none of those scalings gives good fits because all of them make both $B$ and $N_{0}$ - and therefore the radio light curves - to decrease more rapidly than $r^{-1}$ and $r^{-2}$, respectively. This suggests that the index $s$ should be close to our nominal value of $s=1.66$. Since the decline rate of the radio light curves, i.e., their late time emission, is also affected by the parameter $p$, we have investigated models in which both $s$ and $p$ are allowed to vary. This search resulted in much poorer fits than our best one. We acknowledge, though, that the assumption of ad hoc scalings for both $B$ and $N_{0}$ could alleviate these poor fits, at the expense of more complicated models. We defer such detailed study to a further publication. 


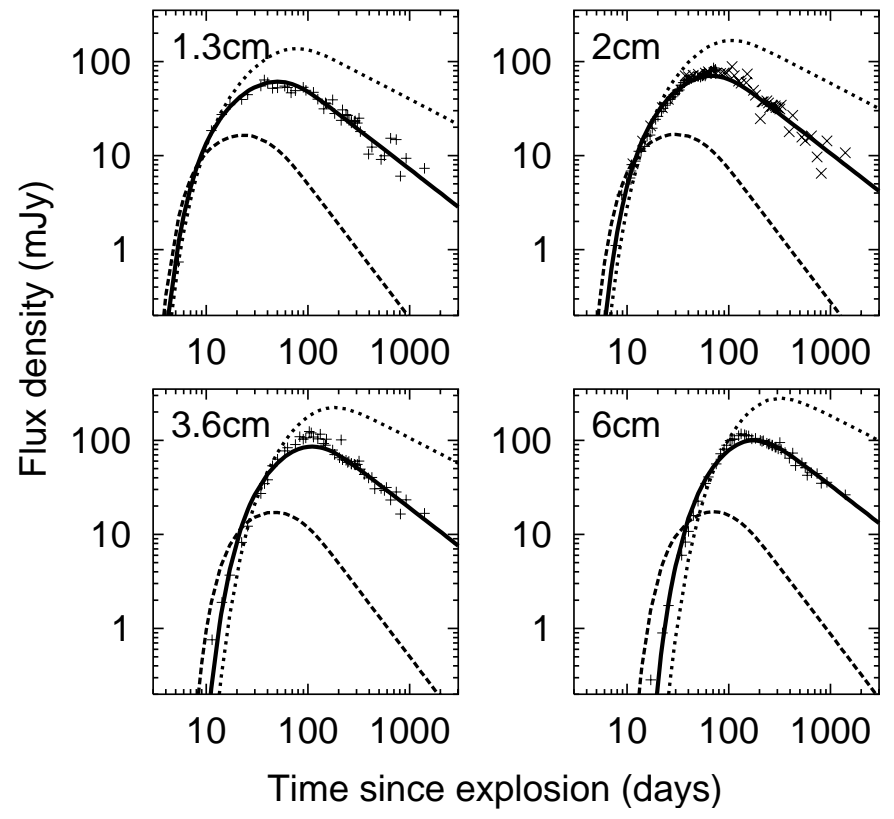

Fig. 3. Observed radio light curves for SN 1993J at 1.3, 2, 3.6, and $6 \mathrm{~cm}$, and model predictions for three different indices of the presupernova circumstellar wind: $s=1.5$ (dotted lines), $s=1.66$ (solid lines), $s=2$ (dashed lines). See text for details.

We investigated as well the effects of using different indices for the expansion $(m)$, but do not find a reasonable fit to the supernova emission using either an undecelerated expansion of the supernova $(m=1)$, or a heavily decelerated expansion $(m=0.75)$.

The unexpected large (initial) magnetic field of about 30G implied by our modeling agrees with the large magnetic field obtained by FB98, and seems to rule out any hypothesis based on a magnetic field due to a compression of the circumstellar wind field. Indeed, since it is very unlikely that the magnetic field energy density in the wind is larger than its kinetic energy density, i.e., $B^{2} / 8 \pi \leq \rho v_{\mathrm{w}}^{2} / 2$, we should expect a magnetic field not in excess of $B \leq 3.4 \mathrm{mG}$ at $r \sim 3 \times 10^{16} \mathrm{~cm}$ (if it arises from a compression of the magnetic field in the wind). This value of the magnetic field is in agreement with the values obtained from polarization observations of $\mathrm{OH}$ masers in supergiants (e.g., Cohen et al. 1987; Nedoluha \& Bowers 1992), which give magnetic field estimates at distances of $\sim 3 \times 10^{16} \mathrm{~cm}$ of $\sim 1-10 \mathrm{mG}$. However, we find that the magnetic field is actually about $0.58 \mathrm{G}$, i.e., 170 times larger. From a strong supernova shock (factor of 4 compression), one would expect an increase of the field value of $\sim 4^{1 / s}$ for a magnetic field that scales as $B \propto r^{-1} \propto n_{\mathrm{sh}}^{1 / s}$, clearly insufficient by almost two orders of magnitude. Therefore, the large magnetic field argues strongly in favor of turbulent amplification in the shell (e.g., Gull 1973; Chevalier 1982a; Chevalier \& Blondin 1995). Furthermore, the assumption that the magnetic energy density evolves as the post-shock thermal energy results in a dependence of the magnetic field with radius of $r^{-1}$. A component of the field behaving like $B \propto r^{-2}$, as suggested from magnetic flux conservation, does not give a reasonably good fit to the observations.

We find that the magnetic energy density in the shell is just a (small) fraction of the post-shock thermal energy density, $\epsilon_{B} / \epsilon_{\mathrm{sh}} \approx 0.024$, but nonetheless much larger than the relativistic electron energy density, $\epsilon_{\mathrm{rel}} / \epsilon_{\mathrm{sh}} \approx$ $2 \times 10^{-4}-10^{-3}$. This points to a strong deviation from equipartition. We note, however, that the ions (whose emission we neglect as it is a factor $m_{\mathrm{p}} / m_{e} \approx 2000$ less than that due to the electrons) might have a large contribution to the total energy density. We also find that the relativistic particle number density is a very small fraction of the particle thermal density. In fact, the ratio $n_{\text {rel }} / n_{\mathrm{sh}}=\left(N_{0} E_{\min }^{1-p} / 2 n_{\mathrm{sh}}\right) \approx(1-8) \times 10^{-7}$ at any given epoch.

Special attention deserves the low-energy cutoff at early epochs that we consider to account for the flattening of the radio light curves at early epochs. We recall that our model assumes an initial injection of (highly) relativistic particles $N(E, r)=N_{0}(r) E^{-p}$, which applies from $E_{\min }$ up to $E_{\max }$. The initial supernova explosion is the likely seed for such a population of relativistic electrons, which must be continuously reaccelerated. We show in Fig. 4 the light curves that we obtain when we do not consider a low-energy cutoff in the relativistic particle distribution, i.e., $\gamma_{\min }=1$. (The other parameters are fixed to their nominal values $p=3, B\left(r_{0}\right)=27 \mathrm{G}$, and $N_{0}\left(r_{0}\right)=6.7 \times 10^{-7} \mathrm{erg}^{p-1} \mathrm{~cm}^{-3}$.) We note that although the optically thin part of the radio light curves remains unaffected, the optically thick part is heavily modified by the lack of a low-energy cutoff. In particular, the radio emission rises more rapidly. More importantly, the observed flattening of the light curves at the shortest wavelengths is not reproduced by such a model. Notice also that at long wavelengths (see the light curve at $\lambda 21 \mathrm{~cm}$ in Fig. 4) the radio emission is strongly absorbed at early times. This is the result of having decreased the minimum energy of the electrons; since the number of relativistic particles is proportional to $E_{\min }^{1-p}$, we effectively increased the column density of the relativistic particles by a large factor. The effect is most easily seen at long wavelengths due to the dependence of the opacity with wavelength $\left(\kappa_{\nu} \propto \lambda^{(p+4) / 2}\right)$. At this point, we also note that our model differs here from that presented by FB98, in that they assume a continuous injection of particles with $\gamma \geq 1$. Bell (1978) showed that particles that are already relativistic can be accelerated in the presence of a shock. Since it is usually assumed that the acceleration of particles in SNe is due to the Fermi process (e.g. Ball \& Kirk 1995 for SN 1986J), we show here that our finding of a low-energy cutoff is consistent with such a process acting in SN1993J. The minimum energy of electrons that can be efficiently accelerated is $\gamma_{\text {min,e }}=\left(m_{\mathrm{p}} / m_{\mathrm{e}}\right)\left(v_{\mathrm{A}} / c \mu\right)$ (Eilek \& Hughes 1991; but see also Ellison et al. 2000, who argue that nonlinear effects in shocks can lead to acceleration of particles from a thermal pool), where $m_{\mathrm{p}}$ and $m_{\mathrm{p}}$ are the protron and electron mass, respectively, $v_{\mathrm{A}}$ is the 


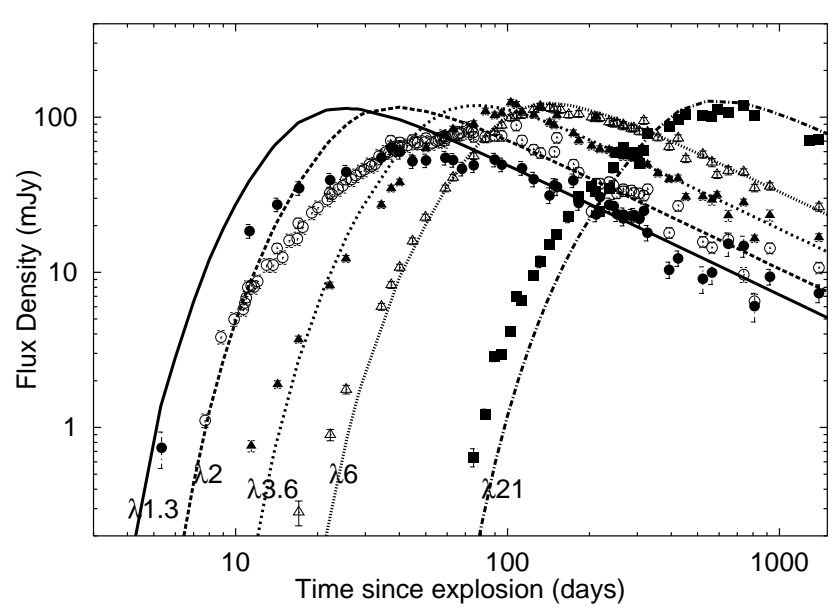

Fig. 4. Radio light curves for SN 1993J. The data is as in Fig. 1. The lines correspond to our best-fit model as described in the text, except that we did not assume a low-energy cut-off. Such model fails to reproduce the flattening of the short-wavelength emission seen at early epochs. See text for details.

Alfvén velocity, $c$ is the speed of light, and $\mu$ is the cosine of the pitch-angle. With the physical conditions deduced for $\mathrm{SN} 1993 \mathrm{~J}, v_{\mathrm{A}}$ varies from $\approx 3200 \mathrm{~km} \mathrm{~s}^{-1}$ at $t=2.3$ days down to about $1200 \mathrm{~km} \mathrm{~s}^{-1}$ at $t=3000$ days. This gives $\gamma_{\text {min }}>20$ and $\gamma_{\text {min }}>7$ at 2.3 and 3000 days, respectively, which is consistent with our results.

It is plausible that Coulomb losses also play a role in the shape of the SN 1993J light curves at high frequencies and early epochs (see FB98, which use power-laws for the circumstellar wind density and the deceleration of the expansion of SN 1993J that are different from the experimental values obtained by Marcaide et al. 1997). However, the neglect of Coulomb losses in our modeling is self-consistent, and does not affect the behavior of the radio light curves either at early or at late epochs. Indeed, the Lorentz factor, $\gamma$, of the electrons responsible for the emission at a given wavelength is $\gamma \approx 84(\lambda B \sin \theta)^{-1 / 2}$ (cgs units), while the Lorentz factor below which Coulomb losses dominate over expansion losses is approximately $\gamma_{\text {Coul }} \approx 180\left(n_{\mathrm{sh}} / 6.9 \times 10^{7} \mathrm{~cm}^{-3}\right)(t / 10 \text { days })^{1-m s}$, where we have neglected for simplicity the slowly-dependent term on the electron energy. From our modeling at $t=$ 10 days, we get $B \approx 7.7 \mathrm{G}$ and $\gamma_{\min } \approx 61$, so the observed emission at $\lambda 1.3 \mathrm{~cm}$ comes mainly from electrons with $\gamma \approx 200$, while Coulomb losses are important for $\gamma \lesssim \gamma_{\text {Coul }} \approx 180$. Analogously, our modeling gives a magnetic field of $\sim 65 \mathrm{mG}$ at current epochs ( $\sim 2700$ days), and $\gamma_{\min } \approx 12$. We then obtain that the observed $\lambda 90 \mathrm{~cm}$ emission comes mainly from electrons with $\gamma \approx 120$, while Coulomb losses are relevant only for electrons with $\gamma \lesssim$ $\gamma_{\text {Coul }} \approx 17$, and thus do not affect the long wavelength radio emission from SN 1993J at late epochs. Therefore, we stress that our main result, namely the dominance of synchrotron self-absorption over external free-free absorption at late epochs is a robust result, and is not affected by the physical details that cause the flattening of the spectra at early epochs.

In summary, we have presented evidence of synchrotron self-absorption for the type II SN 1993J, based on a modeling of its radio light curves. We show that the radio light curves of SN 1993J are better characterized by synchrotron emission that is partially absorbed by a combination of synchrotron self-absorption (inside the shell) and external free-free absorption, rather than only by external free-free absorption, thus confirming previous modeling of the SN 1993J radio light curves (FB98). This result suggests that the "clumpy external absorption" previously invoked to model the radio light curves of SN 1993J might not be needed. We also find that the Razin-Tsytovich effect can be ruled out as well as an alternative mechanism to external free-free absorption. We predict that synchrotron self-absorption is the main absorbing mechanism for the long-wavelength radio emission as SN 1993J ages. This prediction can be tested unambiguously using current technology and observations of SN 1993J at $\lambda 90 \mathrm{~cm}$. Moreover, follow-up observations of SN 1993J at $\lambda \geq 90 \mathrm{~cm}$ are also needed to further constrain the physical parameters of this unique supernova. As pointed out earlier (Chevalier 1998), if SN 1993J is not a bizarre supernova then synchrotron self-absorption is likely to play an important role in most, if not all, radio supernovae, especially as they age.

Acknowledgements. We thank an anonymous referee for useful comments on the manuscript. This work has been partially supported by the Spanish DGICYT Grants No. PB96-0782 and PB97-1164.

\section{References}

Ball, L., \& Kirk, J. G. 1995, A\&A, 303, L57

Bartunov, O. S., Blinnikov, S. I., Pavlyuk, N. N., et al. 1994, A\&A, 281, L53

Bell, A. R. 1978, MNRAS, 182, 147

Carilli, C. L., Perley, R. A., Dreher, et al. 1991, ApJ, 383, 554

Cohen, R. J., Downs, G., Emerson, R., et al. 1987, MNRAS, 225,491

Chevalier, R. A. 1982a, ApJ, 259, 302

Chevalier, R. A. 1982b, ApJ, 258, 790

Chevalier, R. A. 1996, Radio emission from the Stars and the Sun, ed. A. R. Taylor, \& J. M. Paredes, ASP Conf. Ser., Vol. 93, 125

Chevalier, R. A. 1998, ApJ, 499, 810

Chevalier, R. A., \& Blondin, J. M. 1995, ApJ, 442, 312

Eilek, J. A., \& Hughes, P. A. 1991, Beams and Jets in Astrophysics, ed. P. A. Hughes (Cambridge: Cambridge University Press), 428

Ellison, D. C., Berezhko, E. G., \& Baring, M. G. 2000, ApJ, 540,292

Falcke, H. 1996, ApJ, 464, L67

Fillipenko, A. V., \& Matheson, T. 1993, IAU Circ., 5740

Fransson, C., Lundqvist, P., \& Chevalier, R. A. 1996, ApJ, 461, 993 (FLC96)

Fransson, C., \& Björnsson, C.-I. 1998, ApJ, 509, 861 (FB98)

Freedman, W. L., Hughes, S. M., Madore, B. F., et al. 1994, ApJ, 427, 628 
García, F. 1993, IAU Circ., 5731

Gull, S. F. 1973, MNRAS, 162, 135

Lundqvist, P. 1994, in Circumstellar Media in the Late Stages of Stellar Evolution, ed. R. E. S. Clegg, I. R. Stevens, \& W. P. S. Meikle (Cambridge: Cambridge University Press), 213

Marcaide, J. M., Alberdi, A., Ros, E., et al. 1995a, Nature, 373,44

Marcaide, J. M., Alberdi, A., Ros, E., et al. 1995b, Science, 270,1475

Marcaide, J. M., Alberdi, A., Ros, E., et al. 1997, ApJ, 486, L31

Nadyozhin, D. K. 1985, Ap\&SS, 112, 225
Nedoluha, G. E., \& Bowers, P. F. 1992, ApJ, 399, 743

Nomoto, K., Suzuki, T., Shigeyama, T., et al. 1993, Nature, 364,507

Pérez-Torres, M. A. 1999, Ph.D. Thesis, University of Valencia, Spain

Pooley, G. G., \& Green D. A. 1993, MNRAS, 264, L17

Podsiadlowski, Ph., Hsu, J. J. L., Joss, P. C., et al. 1993, Nature, 364, 509

Van Dyk, S. D., Weiler, K. W., Sramek, R. A., et al. 1994, ApJ, 432, L115

Weiler, K. W., Panagia, N., Sramek, R. A., et al. 1989, ApJ, 336,421

Weiler, K. W., Panagia, N., \& Sramek., R. A. 1990, ApJ, 364, 611 